\title{
Inhaltsverzeiehnis.
}

\section{Allgemeiner Teil.}

1. Litteraturgeschichte. Von Dr. Otto Harnack, Professor an der Technischen Hochschule zu Darmstadt.

2. Geschichte der deutschen Philologie. Von Dr. Wolfgang Golther, Professor an der Universität Rostock.

3. Schrift- und Buchwesen. Von Dr. Oskar von Hase in Leipzig.

4. Kulturgeschichte. Von Dr. Georg Liebe, Assistenten am Staatsarchiv zu Magdeburg.

5. Volkskunde. Von Dr. Adolf Hauffen, Professor an der Universität Prag.

6. Die Litteratur in der Schule. Von Dr. Ernst Naumann, Professor am Friedrich Wilhelms-Gymnasium zu Berlin.

7. Geschichte der neuhochdeutschen Schriftsprache. Von Dr. Willy Scheel in Berlin.

8. Geschichte der Metrik. 1893, 1894. Von Dr. Jakob Minor, Professor an der Universität Wien.

9. Kunstgeschichte. Von Dr. Cornelius Gurlitt, Professor an der Technischen Hochschule zu Dresden.

10. Musikgeschichte. Von Professor Dr. Heinrich Reimann, Bibliothekar an der Königlichen Bibliothek zu Berlin.

11. S toffgeschichte. Von Dr. Johannes Bolte, Oberlehrer am Königstädtischen Gymnasium zu Berlin.

12. Geschichte des Unterrichts- und Erziehungswesens. Von Professor Dr. Karl Kehrbach in Berlin.

13. Poetik und ihre Geschichte. Von Dr. Richard Maria Werner, Professor an der Universität Lemberg. Vgl. Ba. 6 der JBL.

\section{Von der Mitte des 15. bis zum Anfang des 17. Jahrhunderts.}

1. Allgemeines. Von Dr. Max Osborn in Berlin.

2. Lyrik. Von Dr. Georg Ellinger, Oberlehrer an der 6. Städtischen Realschule zu Berlin.

3. Epos. Von Dr. Adolf Hauffen, Professor an der Universität Prag.

4a. Drama. Von Dr. Wilhelm Creizenach, Professor an der Universität Krakau.

b. Hans Sachs. Von Dr. Karl Drescher, Privatdocenten an der Universität Bonn.

כ. Didaktik. 1893, 1894. Von Dr. Adolph Hofmeister, Kustos der Universitätsbibliothek zu Rostock.

6. Luther und die Reformation. Von Dr. Gustav Kawerau, Professor an der Universität Breslau.

7. Humanisten und Neulateiner. Von Dr. Georg Ellinger, Oberlehrer an der 6. Städtischen Realschule zu Berlin. 


\section{Vom Anfang des 17. bis zur Mitte des 18. Jahrhunderts.}

1. Allgemeines. Von Dr. Alexander Reifferscheid, Professor an der Universität Greifswald.

2. Lyrik. Von Dr. Ludwig Pariser in München.

3. Epos. Von Dr. Alexander Reifferscheid, Professor an der Universität Greifswald.

4. Drama. Von Dr. Johannes Bolte, Oberlehrer am Königstädtischen Gymnasium zu Berlin.

5. Didaktik. Von Dr. Victor Michels, Professor an der Universität Jena.

\section{Von der Mitte des 18. Jahrhunderts bis zur Gegenwart.}

1. Allgemeines.

a) Litteraturgeschichte. Von Dr. Adolf Stern, Professor an der Technischen Hochschule zu Dresden.

b) Politische Geschichte. 1893, 1894. Von Dr. Georg Winter, Archivar am Staatsarchiv zu Stettin.

c) Memoiren, Tagebücher und Briefwechsel. Von Dr. Franz Muncker, Professor an der Universität München.

d) Die deutsche Litteratur und das Ausland. Von Dr. Adolf Stern, Professor an der Technischen Hochschule zu Dresden.

2. Lyrik.

a) Von der Mittedes 18. Jahrhunderts bis zu den Freiheitskriegen. Von Dr. August Sauer, Professor an der Universität Prag.

b) Von den Freiheitskriegen bis zur Gegenwart. Von Dr. Julius Elias in Berlin.

3. Epos. 1893, 1894. Von Dr. Richard Rosenbaum in Berlin.

4. Drama und Theatergeschichte. Von Dr. Alexander von Weilen, Privatdocenten an der Universität Wien. Vgl. Bd. 6 der JBL.

5. Didaktik. Von Dr. Richard M. Meyer, Privatdocenten an der Universität Berlin.

6. Lessing. Von Dr. Erich Schmidt, Professor an der Universität Berlin.

7. Herder. Von Dr. Ernst Naumann, Professor am Friedrich-WilhelmsGymnasium zu Berlin.

8. Goethe.

a) Allgemeines. Von Professor Dr. Veit Valentin in Frankfurt a. M.

b) Leben. Von Dr. Karl Heinemann, Oberlehrer am Kgl. Gymnasium zu Leipzig.

c) Lyrik. Von Dr. Otto Pniower in Berlin.

d) Epos. Von Dr. Georg Witkowski, Professor an der Universität Leipzig.

e) Drama. Von Dr. Georg Witkowski, Professor an der Universität Leipzig.

9. Schiller. Von Dr. Albert Köster, Professor an der Universität Marburg.

10. Romantik. Von Dr. Oskar F. Walzel, Privatdocenten an der Universität Wien.

11. Das junge Deutschland. Von Dr. Ernst Elster, Professor an der Universität Leipzig. $\mathbf{V}_{\text {gl. Bd. }} 6$ der JBL.

Autorenregister.

Sachregister.

Siglenregister.

Bemerkungen für den Gebrauch.

Druckfehlerverzeichnis.

Danktafel. 